\title{
On the onset of recurrent eruptions of a filament observed during August 2012
}

\author{
Nandita Srivastava $^{1}$, Anand D. Joshi ${ }^{2}$ and Shibu K. Mathew ${ }^{1}$ \\ ${ }^{1}$ Udaipur Solar Observatory, Physical Research Laboratory, Udaipur, India. \\ email: nandita@prl.res.in; shibu@prl.res.in \\ ${ }^{2}$ Korea Astronomy and Space Science Institute (KASI), Daejeon, Korea \\ email: anand@kasi.re.kr
}

\begin{abstract}
We report observations of a long filament that underwent recurrent partial eruptions on August 4, 6, and 8, 2012. The filament reappeared in the subsequent rotation of the Sun, and disappeared completely on August 31, 2012. We implemented an automated filament detection algorithm developed by us for estimating different attributes of these filaments few hours prior to its disappearance in $\mathrm{H} \alpha$ and studied their evolution. Based on these attributes, we determine the onset time of the disappearance of $\mathrm{H} \alpha$ filaments. We then compared these onset times with that of the associated CMEs observed by LASCO/SOHO coronagraphs. This is also useful to understand temporal relationship of EUV and X-ray flux variation associated with filament disappearances in $\mathrm{H} \alpha$. Our results show the importance of such studies in understanding the mechanism of CME initiation, particularly the role of eruptive filaments, in this process.
\end{abstract}

Keywords. Filaments, CMEs, Onset time, etc.

\section{Introduction}

Coronal Mass Ejections (CMEs) are the key drivers of space weather and are known to be the main cause of major geomagnetic storms at the Earth (Gosling, 1993). They are often associated with flares and eruptive filaments or EFs (Webb et al. 1976; Webb and Hundhausen, 1987). Munro et al. (1979) found that more than $70 \%$ of the CMEs were associated with EFs. Those originating from around centre of solar disc are potential candidates for geo-effective CMEs as shown by Srivastava \& Venkatakrishnan (2004). CMEs associated with flares and eruptive prominences differ in their properties. A recent study by Joshi and Srivastava (2011) has shown that flare associated CMEs show bimodal acceleration while filament associated CMEs do not. As per our present understanding, same mechanism drives all CMEs, and the two types of CMEs, lie at the two extremes of the energy range that is driving them. One of the major constraints in investigating the driving force of CMEs is the difficulty in estimating their correct onset time. The onset time is currently estimated by back extrapolating the projected height-time plot of the leading edge of a CME using LASCO/SoHO observations (http://cdaw.gsfc.nasa.gov). In the events where the source region involves a filament, monitoring its activation is crucial for forewarning of its disappearance in $\mathrm{H} \alpha$, in EUV and also the associated soft X-ray (SXR) flare and CME lift off. Determining precise temporal relationship between the eruption of $\mathrm{H} \alpha$ and EUV filament, SXR emission and appearance of CME leading edge (in white light) is therefore of utmost importance in understanding the CME initiation mechanism. To achieve this objective, we examine, recurrent eruptions of a single large filament and the associated CMEs that occurred on August 4, 6, 8 and in the next rotation of the sun on August 31, 2012. 


\section{Events, Analysis and Summary}

A long filament appeared on the Sun on August 1 at the SE limb as observed in $\mathrm{H} \alpha$ images. On August 4, it showed activations leading to its disappearance in $\mathrm{H} \alpha$ at around 12:20 UT. This was associated with a C3.5 class flare observed by GOES and enhancement in EUV flux observed by LYRA aboard PROBA2 (Dominique et al. 2013). The filament eruption was associated with a white light halo CME with projected speed of $850 \mathrm{~km} / \mathrm{s}$ as observed by LASCO-C2 coronagraph at 13:22 UT. Following this, the filament reformed and underwent subsequent eruptions on August 6 and 8 accompanied by C-class flares and slow and accelerating CMEs. It may be noted that all the three eruptions were not powerful enough to drive a geomagnetic storm although the location of the filaments was favourable (Srivastava and Venkatakrishnan, 2004). In the next rotation of the Sun, the filament reappeared on August 30, on the SE limb and erupted completely on August 31 at 19:40 UT in $\mathrm{H} \alpha$ with an associated C8.4 class flare and EUV flux peaking around 20:43 UT and 21:13 UT. Concurrently, LASCO-C2 observed a partial halo CME with a speed of $1440 \mathrm{~km} / \mathrm{s}$. Although this CME was in SE direction, it gave rise to a moderate geomagnetic storm on September 3 (Dst $\sim-78 \mathrm{nT}$ ). As described above, the chain of recurrent eruptions of a long quiescent filament at intervals of few days, provides an excellent data-set to understand the initiation, and propagation of slowly accelerating CMEs during the minimum phase of the solar cycle, which under suitable circumstances may prove to be geoeffective. We implemented an algorithm developed by Joshi, Srivastava and Mathew (2010) on the full disk $\mathrm{H} \alpha$ images obtained for all the 4 events. This algorithm detects, tracks and estimates the location, length, area and number of fragments of disappearing filaments. From the temporal evolution of these attributes, we estimated the time of start and end of $\mathrm{H} \alpha$ filament disappearance, compared these with the time of SXR flux peak, EUV flux peak and CME lift off. Our analysis shows that the start time of the filament disappearance in $\mathrm{H} \alpha$ precedes the time of peak of SXR flux by more than $1.5 \mathrm{hr}$. The EUV flux peaks 15 to $30 \mathrm{~min}$ after the SXR flux. Further, the $\mathrm{H} \alpha$ filament disappearance starts at least an hour earlier than the onset time of CME. These clearly demonstrate the usefulness of implementation of the automated filament detection technique to forewarn potential geo-effective eruptions, based on full disk $\mathrm{H} \alpha$ observations, prior to the launch of a CME.

\section{Acknowledgements}

This work contributes to the research for European Union Seventh Framework Programme (FP7/2007-2013) for the COMESEP project under Grant Agreement No. 263252. We thank the GONG \& PROBA2 team for providing $\mathrm{H} \alpha$ and LYRA data, respectively.

\section{References}

Dominique, M., Hochedez, J.-F., Schmutz, W., Dammasch, I. E., Shapiro, A. I., Kretzschmar, M., Zhukov, A. N., Gillotay, D. et al. 2013, Solar Phys., 286,1, 21-42

Gosling, J. T. J. Geophys. Res, 98, A11, 18937-18950

Joshi, A. D., Srivastava, N., \& Mathew, S. K. 2010, Solar Phys., 262, 425-436

Joshi, A. D. \& Srivastava, N. 2011, Astrophys. J.,739, 1, 8

Munro, R. H., Gosling, J. T., Hildner, E., MacQueen, R. M., Poland, A. I., Ross, C. L.1979, Solar Phys. 61, 201-215

Srivastava, N. \& Venkatakrishnan, P.2004, J. Geophys. Res. 109, A10, CiteID A10103

Webb, D. F., Krieger, A. S., \& Rust, D. M. 1976, Solar Phys. 48, 159-186

Webb, D. F. \& Hundhausen, A. 1987, Solar Phys.108, 2, 383-401. 